\title{
CARACTERIZAÇÃO FíSICO-QUÍMICA DE BATATA-DOCE (Ipomoea batatas L.) COMUM E BIOFORTIFICADA
}

\author{
Diandra Carvalho de Sá Nolêto ${ }^{1}$, Camila Raia Pereira da Silva1, Charllyton Luis Sena da Costa1, Valdiléia Teixeira \\ Uchôa ${ }^{2^{*}}$
}

\author{
${ }^{1}$ Departamento de Nutrição, Faculdade Santo Agostinho, Av. Valter Alencar 665, São Pedro, Teresina - PI. CEP: 64019-625. \\ 2*Departamento de Química, Universidade Estadual do Piauí, R. João Cabral, 2231, Pirajá, Teresina - PI, CEP: 64002-150.
}

$2^{*}$ Autor para correspondência: vtuquimica@yahoo.com.br

RESUMO: Compararam-se os teores de betacaroteno, carboidratos e lipídios presentes na batata-doce biofortificada e na batata-doce comum por meio da sua caracterização físico-química. Foram realizadas análises de carotenoides, para o composto orgânico $\beta$-caroteno, além de pH, lipídeos e carboidratos. De acordo com os resultados obtidos, observou-se que a batata-doce comum apresentou teores insignificantes de carotenoides devido à ausência de formação de banda no comprimento de onda entre 400 e $500 \mathrm{~nm}$, enquanto que a batata biofortificada apresentou banda característica da presença de carotenoides em torno de $450 \mathrm{~nm}$. Os valores de $\mathrm{pH}$ encontrados para ambas as batatas-doces foram na faixa de 6 e 7. Quanto aos macronutrientes, a batata-doce biofortificada revelou maior teor de lipídeos (1,09\%) quando comparado à comum (0,94\%). Em relação aos carboidratos, observou-se o inverso, em que a batata-doce comum apresentou conteúdo de 15,6\%, enquanto a biofortificada $13,58 \%$. Assim, a batata-doce biofortificada é uma alternativa no combate e controle dos casos de hipovitaminose $A$, por seu alto teor de $\beta$-caroteno, precursor da vitamina $A$, produto agrícola de boa aceitabilidade e acessível às populações carentes que são os grupos mais vulneráveis para deficiências de micronutrientes.

PALAVRAS-CHAVE: Biofortificação, $\beta$-caroteno, Batata-doce.

\section{PHYSICO-CHEMICAL CHARACTERIZATION OF SWEET POTATO (Ipomoea batatas) AND COMMON BIOFORTIFIED}

\begin{abstract}
They compared the beta-carotene content, carbohydrates and lipids present in sweet potato biofortified and common sweet potato, through its physicochemical characterization. Carotenoid analyzes were performed for $\beta$-carotene organic compound, as well as $\mathrm{pH}$, lipids and carbohydrates. According to the results, it was observed that the common sweet potatoes showed insignificant levels of carotenoids due to the absence band formation at a wavelength between 400 and $500 \mathrm{~nm}$, while the potato biofortified showed characteristic band in presence of carotenoids about $450 \mathrm{~nm}$. The $\mathrm{pH}$ values found for both sweet potatoes. Concerning macronutrients, showed a higher content of lipids in biofortified sweet potatoes were in the range of 6 and 7 . As for macronutrients, the sweet potato biofortified revealed higher content of lipids (1.09\%) when compared to common (0.94\%). Regarding the carbohydrates, we observed the opposite, in that the common sweet potato presented content of $15.6 \%$, while the biofortified $13.58 \%$. Thus, the sweet potato biofortified is an alternative in the fight and control of cases of vitamin A deficiency, for its high content of $\beta$-carotene, vitamin A control of cases of vitamin A deficiency, for its high content of $\beta$-carotene, vitamin A precursor, agricultural product of good acceptability and accessible to poor populations are the most vulnerable groups to micronutrient deficiencies.
\end{abstract}

KEYWORDS: Biofortification, $\beta$-carotene, sweet potato. 


\section{INTRODUÇÃO}

A batata-doce (Ipomoea batatas L.) é originária das Américas Central e do Sul, sendo encontrada desde a Península de Yucatam, no México, até a Colômbia. Relatos de seu uso remontam de mais de dez mil anos, com base em análise de batatas secas encontradas em cavernas localizadas no vale de Chilca Canyon, no Peru e em evidências contidas em escritos arqueológicos encontrados na região ocupada pelos Maias, na América Central (Silva, 2004).

Por ser uma raiz muito presente na dieta das populações, a batata-doce é um bom meio para melhorar a nutrição dessas pessoas (Silva et al., 2010). As variedades de batata-doce comercializadas no Brasil são predominantemente, de cor de polpa amarela, branca e creme. Raramente, são encontradas batatasdoces de polpa alaranjadas ricas em carotenoides (Melo et al., 2010).

Uma nova alternativa de combate a deficiências de micronutrientes vem sendo desenvolvida no Brasil pela Empresa Brasileira de Pesquisa Agropecuária - EMBRAPA, aquela consiste na biofortificação de produtos como, batata-doce, feijão, milho e mandioca com maiores teores de pró-vitamina $A$, ferro e zinco (Silva, 2008).

Segundo Hotz e McClafferty (2007), a biofortificação oferece modificações nos vegetais, como intervenção de saúde pública, e pode tornar-se uma ação efetiva para a população rural (composta principalmente por indivíduos com maiores riscos de apresentar deficiências de micronutrientes). Como os vegetais biofortificados são fontes alimentares viáveis no tratamento de carências nutricionais como ferro, zinco e vitamina $A, 0$ acesso a essas cultivares, seria uma alternativa para o fornecimento de alimentos mais nutritivos, que podem ajudar a reverter à carência de nutrientes, e, assim, reduzir o número de casos de desnutrição infantil no Brasil (Stevens e Winternelson, 2008).

No Brasil, as atividades dos projetos de biofortificação HarvestPlus (apoiado pela Fundação Bill e Melinda Gates e pelo Banco Mundial, entre outros), AgroSalud (que conta com o apoio da Agência Canadense para o Desenvolvimento Internacional CIDA) e BioFORT (que conta com apoio do Fundo de Pesquisa EMBRAPA-MONSANTO) são coordenadas pela Embrapa, onde são pesquisados algumas cultivares como o arroz, o feijão, a batata-doce, a mandioca, o milho, o feijão caupi, o trigo e a abóbora (Carvalho e Nutti, 2012).

Segundo Rodriguez-Amaya (2008), a utilização de batata-doce de polpa alaranjada se apresenta como alternativa para a suplementação alimentar por ser uma fonte barata e abundante de $\beta$-caroteno, e assim, suprir a carência de vitamina A. Um indicador de uma maior concentração de $\beta$-caroteno, precursor de vitamina $A$, é a variação de sua coloração interna, isto é, quanto mais escura for a tonalidade, maior será a concentração de $\beta$-caroteno (Mirasse, 2010).

No Brasil, a cultivar de batata-doce alaranjada Beauregard foi selecionada pela EMBRAPA e as avaliações do germoplasma introduzidas foram realizadas nos seguintes Estados: Minas Gerais, Maranhão, Sergipe, Distrito Federal e Goiás, considerando o teor de betacaroteno, a produtividade e a aceitação por consumidores (Alves et al., 2012).

A batata-doce Beauregard é uma cultivar americana desenvolvida em 1981 pela Louisiana Agricultural Experiment Satation (Embrapa, 2010). Apresenta em média $115 \mu \mathrm{g}$ de $\beta$-caroteno/g de raiz fresca (185 $\mu \mathrm{g} . \mathrm{g}-1$ de raiz fresca de carotenóides totais) e comparado à batata-doce, a quantidade de $\beta$-caroteno varia de $<1 \mu \mathrm{g} . \mathrm{g}-1$ (raiz branca) a $130 \mu \mathrm{g} . \mathrm{g}-1$ de raiz fresca (Rodrigues-Amaya et al., 2011). Berni et al. (2014), afirmam que as batatasdoces de polpa laranja são capazes de suprir até 3,6 vezes as quantidades diárias recomendadas para a ingestão de vitamina $A$.

Diante do que foi exposto, esta pesquisa teve por objetivo comparar os teores de betacaroteno e macronutrientes presentes na batata-doce biofortificada e na batata-doce comum, através da sua caracterização físico-química.

\section{MATERIAL E MÉTODOS}

\section{Amostras}

As amostras de batata-doce de polpa alaranjada foram obtidas da horta comunitária da Fazenda da Paz com escritório localizado na Rua São Pedro no Centro de Teresina - PI e as de batata- 
doce comum de comércio localizado na Avenida Prof Valter Alencar na cidade de Teresina-PI entre outubro de 2014 e abril de 2015.

\section{Análise de espectroscopia de absorção no Ultra Violeta-Visível (UV-Visível)}

Realizou-se a análise de betacaroteno por espectroscopia de absorção no UV-visível no Laboratório de Química da Universidade Federal do Piauí (UFPI). Para a amostra de betacaroteno foram pesados, em balança analítica da marca Bel, $10 \mathrm{~g}$ de batata-doce comum triturada e transferidos para erlenmeyer de $125 \mathrm{~mL}$ e então, $100 \mathrm{~mL}$ de hexano grau analítico PA da marca Qeel foram depositados sobre a amostra e deixados em contato por 3 horas. 0 procedimento foi realizado em triplicata e reproduzido para as amostras de batata-doce biofortificada.

Os extratos hexânicos filtrados foram analisados por espectroscopia de absorção molecular no UV-Visível utilizando um espectrofotômetro duplo feixe Lambda 25 da Perkin Elmer. Foi realizada uma varredura espectral para cada uma das amostras utilizando cubetas de quartzo de $1 \mathrm{~cm}$ de caminho óptico. As leituras foram realizadas entre 190 - 800 $\mathrm{nm}$ e os espectros de absorção resultantes foram então registrados.

\section{Análise de espectroscopia de absorção molecular no Infravermelho Médio}

Analisaram-se carotenoides presentes na batata-doce biofortificada por espectroscopia de absorção molecular no Infravermelho médio no Laboratório de Química Orgânica da Universidade Estadual do Piauí (UESPI), no qual se extraiu a fração apolar (compostos orgânicos com caráter químico apolar) das batatas (biofortificada e comum) com hexano $(200 \mathrm{~mL})$, grau analítico. Produziram-se pastilhas contendo $100 \mathrm{mg}$ de Brometo de potássio $(\mathrm{KBr})$ e 0,2 $\mathrm{mL}$ do extrato hexânico obtidos da batata biofortificada e batata comum. Analisaramse as pastilhas obtidas num espectrofotômetro de Infravermelho IRAffinity ${ }^{\circledR}$ Shimadzu na faixa de 4000 a $400 \mathrm{~cm}^{-1}$, equipado com software de aquisição e análise IRsolution $囚$. Os espectros obtidos representam a média de 64 varreduras espectrais, na faixa de trabalho.

\section{Análises Físico-Químicas}

As análises do $\mathrm{pH}$, glicídios e lipídeos, foram determinadas em triplicata, seguindo a metodologia do Instituto Adolfo Lutz (1985) e foram realizadas no Laboratório de Bromatologia da Faculdade Santo Agostinho (FSA).

\section{Análise Estatística}

O software GraphPad Prism 5.0 foi utilizado para as análises estatísticas, sendo os dados avaliados pelo Teste t-Student através de médias e desvio-padrão.

\section{RESULTADOS E DISCUSSÃO}

\section{Determinação de Betacaroteno presente nas amostras de Batata Doce Comum e Biofortificada através de Espectrofotometria em UV-Visível}

$\mathrm{Na}$ análise da Figura $1 \mathrm{~A}$, referente ao extrato hexânico da batata comum, observou-se a ausência de formação de banda de comprimento de onda. Isto significa que este extrato não possui nenhuma substância orgânica com ligações dupla conjugada, denominado cromóforo, que caracteriza a presença do composto orgânico $\beta$-caroteno. Portanto, a baixa absorção de luz pelo espectro da batata-doce comum revelou teores insignificantes de carotenoides. Por outro lado, os espectros da batata biofortificada apresentaram a absorção de luz de alta intensidade, devido a existência de ligações duplas conjugadas, caracterizando da presença de $\beta$-caroteno entre 400 - 500 nm (Figura 1-B). Diante disso, constatou-se a sobreposição da batata-doce biofortificada em relação à comum, é em relação ao carotenoide em estudo.

A Figura 2 mostra os resultados encontrados no UV-Visível, para efeito de interpretação dos dados utilizou-se o Teste $t$ de student, para a comparação das médias entre as amostras, utilizando-se um nível de significância de 5\%. Após a aplicação do Teste t, observou-se que existe diferença significativa entre as batatas quanto ao teor de caroteno, já que apresentou valores de $p<0,05$. 
Figura 1. (A)- Espectros de absorção no UV-Visível dos extratos de batata-doce comum entre $320-800 \mathrm{~nm}$. (B)Espectros de absorção no UV-Visível dos extratos de batata-doce biofortificada entre 320 - $800 \mathrm{~nm}$.

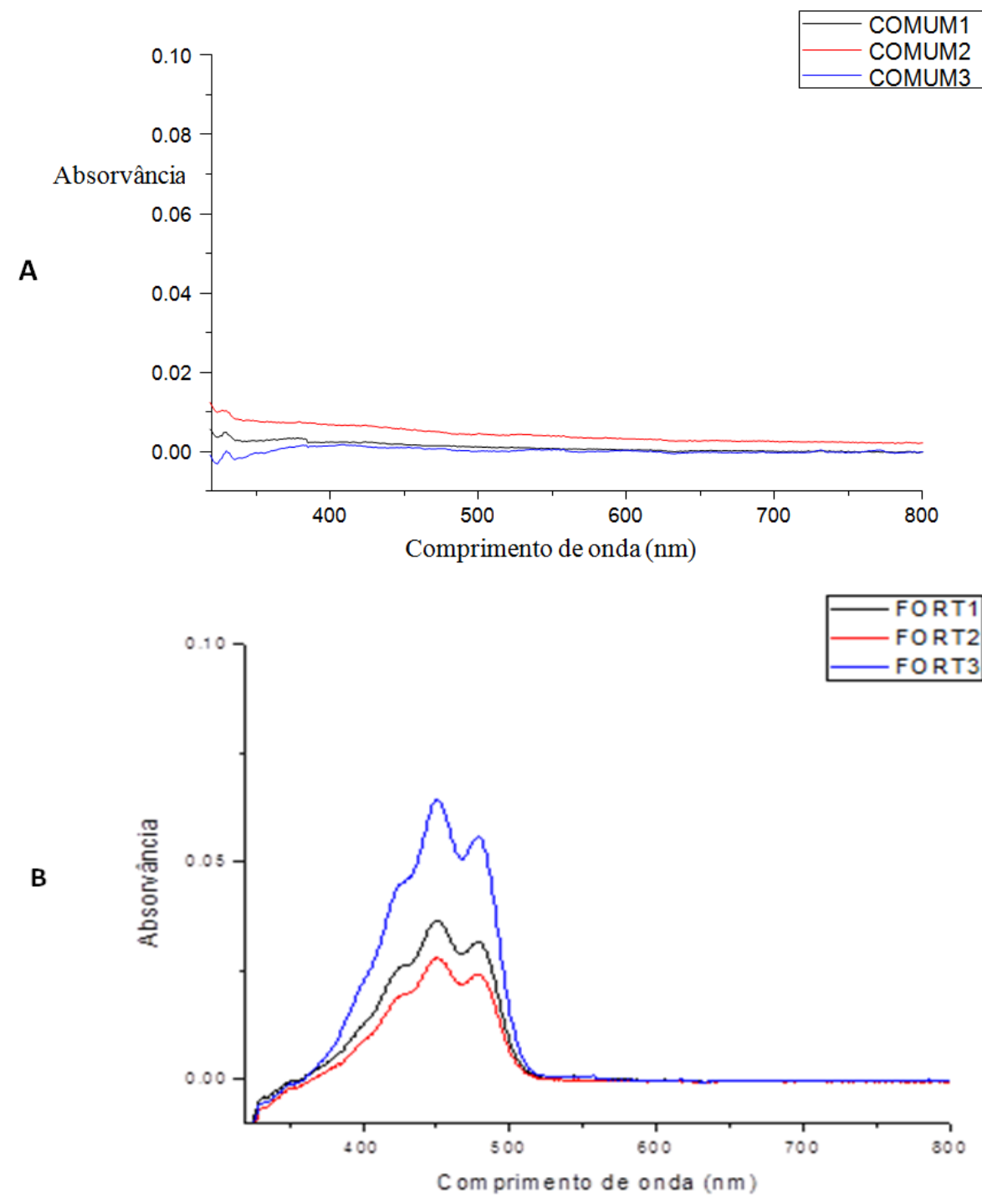

Figura 2. Níveis de carotenoides presentes nas diferentes cultivares de batata-doce medidas por UV-Visível e expressas como áreas dos sinais de carotenoides integradas

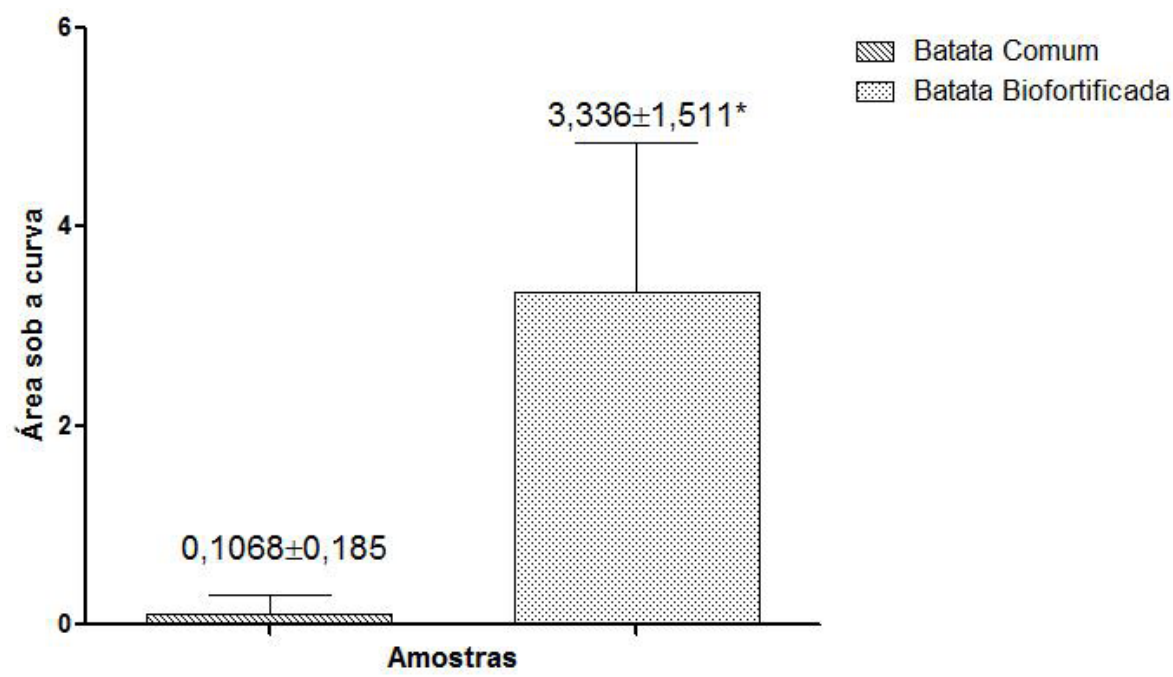


De acordo com Silva et al. (2008), as variedades de batata-doce de polpa branca apresentam teores insignificantes de caroteno, enquanto que as variedades de polpa alaranjada contêm valores superiores aos encontrados na cenoura, que é o alimento mais mencionado como fonte de caroteno, mostrando relação direta com os resultados encontrados.

Em estudos feitos com cenouras, observouse uma banda com $\lambda$ máx $460 \mathrm{~nm}$, caracterizando duplas ligações conjugadas do betacaroteno (Zeraik e Yariwake, 2008), com valores próximos aos encontrados na batata doce biofortificada $\sim 450 \mathrm{~nm}$.

De acordo com Embrapa (2010), a cultivar Beauregard possui raízes de polpa alaranjada-intenso mostrando ser mais rica em $\beta$-caroteno, podendo esta hortaliça ser uma alternativa de merenda escolar com alto valor nutricional (Nunes et al., 2012).

Segundo Britton (1995), a maior parte dos carotenoides pode absorver três comprimentos de onda, no máximo, gerando três espectros de pico. 0 licopeno de coloração avermelhada absorve 0 mais longo comprimento de onda ( $\lambda \max , 444,470$ e 502 nm), com 11 ligações duplas conjugadas em sua estrutura (Figura 3). E o bicíclico $\beta$-caroteno, embora apresente o mesmo número de ligações duplas como licopeno, é amarelo alaranjado e tem $\lambda$ max a 450 entre $477 \mathrm{~nm}$, faixa equivalente à encontrada na batata-doce alaranjada em estudo.

Figura 3. Espectro de absorção molecular na região do infravermelho do extrato hexânico da batata-doce biofortificada

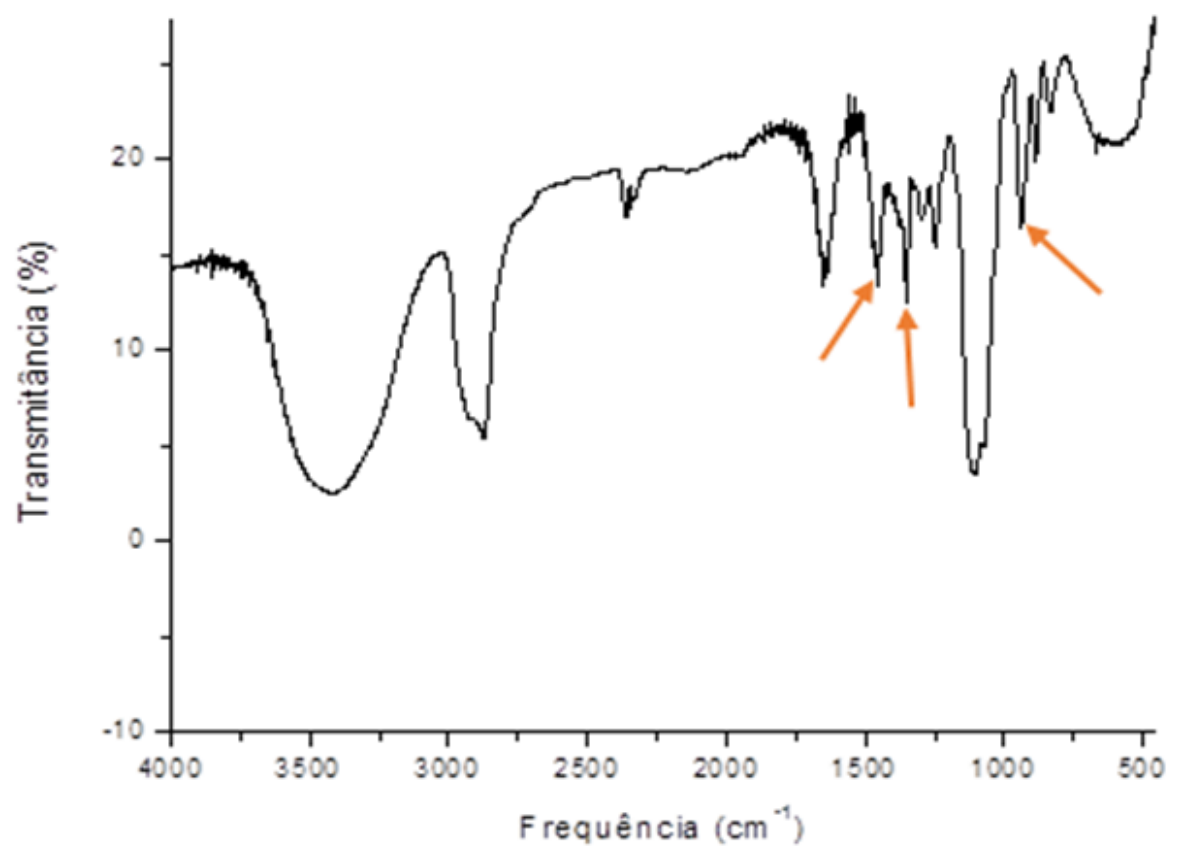

Identificação do carotenoide presente na batatadoce biofortificada por espectroscopia no infravermelho

A espectroscopia de absorção molecular na região do Infravermelho médio é uma técnica analítica consagrada para análises qualitativas de diferentes amostras orgânicas, fundamenta-se na absorção diferencial da radiação eletromagnética por parte de moléculas orgânicas. A absorção ocorre de maneiras distintas dentro de uma mesma molécula onde cada fragmento molecular pode reagir de maneira diferente. Desta forma, o espectro de absorção no infravermelho resultante da análise de uma determinada molécula representa uma medida de seus elementos constitucionais característicos, conduzindo à identificação da molécula (Pavia et al., 2010).

0 espectro de absorção no infravermelho da amostra de batata-doce biofortificada (Figura 3) apresentou sinais característicos da estrutura do $\beta$-caroteno. Os sinais presentes, indicados pelas setas, na faixa compreendida entre $1458-1350 \mathrm{~cm}^{-1}$ são característicos das deformações associadas aos grupos $\mathrm{CH}_{2}$ e $\mathrm{CH}_{3}$ da estrutura do carotenoide. $\mathrm{O}$ sinal observado em $937 \mathrm{~cm}^{-1}$ decorre da deformação angular fora do plano das ligações $\mathrm{CH}$ do sistema de ligações duplas conjugadas em configuração trans 
característico da molécula do $\beta$-caroteno (Baranska et al., 2006; Ammawath e Man, 2010).

Considerando-se as evidências espectroscópicas apresentadas acima é possível afirmar que a batata-doce biofortificada apresenta 0 $\beta$-caroteno (Figura 3) como agente pigmentante em sua composição, o que aumenta o potencial nutricional, do material já que o $\beta$-caroteno é o mais eficiente dos carotenoides para a síntese de vitamina $A$.

\section{Caracterização química de Lipídeos e Carboidratos}

Os carotenoides fazem parte do grupo dos lipídeos, o que explica sua baixa solubilidade em água, mas bastante solúvel em solventes orgânicos como éter e hexano. Consequentemente, sua maior concentração na batata doce biofortificada contribui diretamente no aumento dos níveis de lipídeos (Figura 4). 0 presente estudo revelou maior teor de lipídeos na batata-doce biofortificada $(1,09 \%)$ quando comparado à comum $(0,94 \%)$, satisfazendo a afirmação acima. Nascimento (2013) analisando $100 \mathrm{~g}$ de fécula de batata-doce biofortificada, encontrou valores de $0,6 \%$ de lipídeos. Isso, pode indica que o processo de transformação da batatadoce biofortificada em fécula, pode levar a uma perda no teor de lipídeos.

Figura 4. Teor de lipídeos através de média e desvio-padrão das amostras analisadas de batata-doce comum e biofortificada.

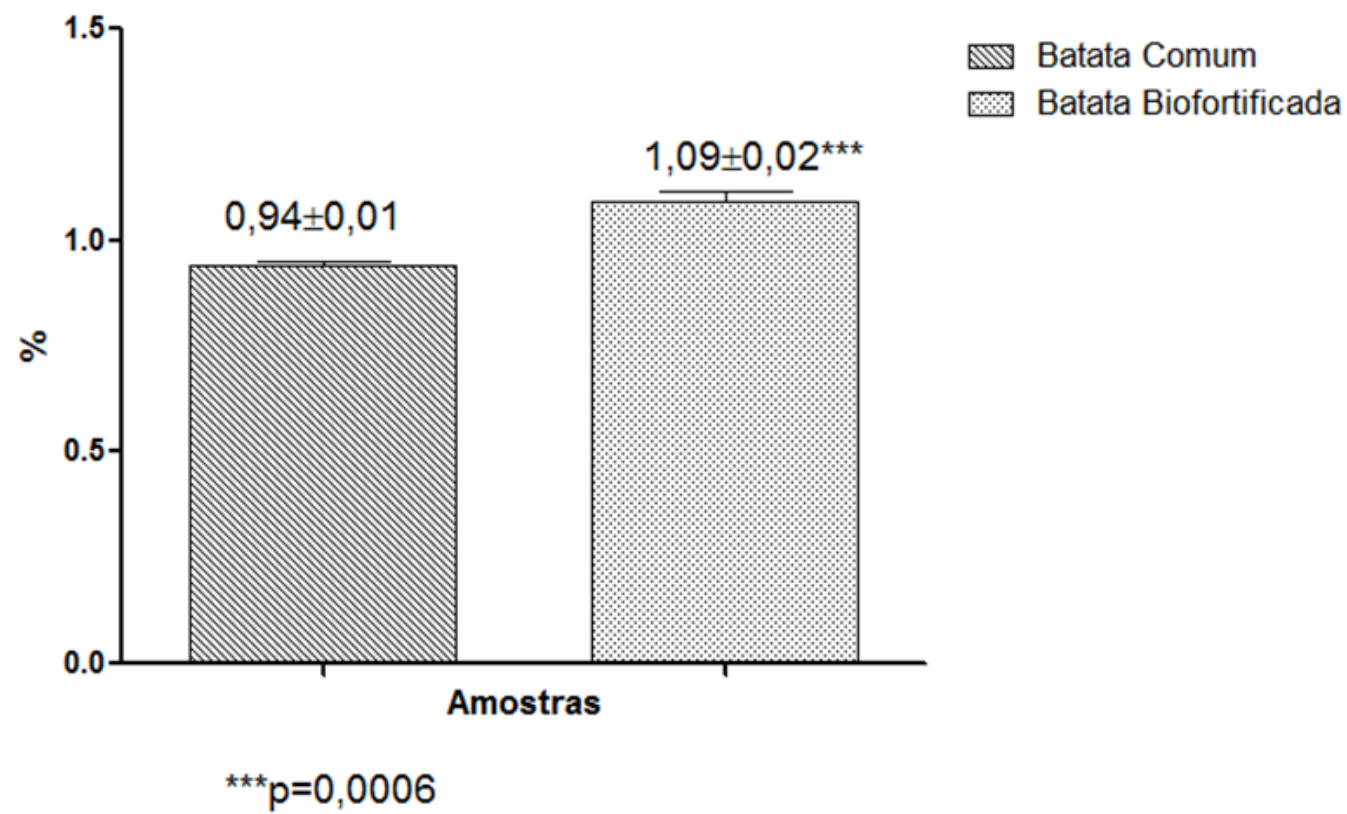

Sobre o teor de lipídeos encontrados na literatura, para os tubérculos in natura são mostrados dados da batata-doce comum com teores lipídicos entre $0,3 \%$ a $0,8 \%$ (Freitas et al., 2007), valores superiores aos encontrados por Leonel e Cereda (2000) com percentuais lipídicos em torno de 0,15 e $0,14 \%$, respectivamente. Já a batata-doce biofortificada apresentou teor de lipídeos de 0,45\%.

Os resultados obtidos neste estudo, em relação ao teor de lipídeos, também foram superiores aos verificados por Ahmed et al. (2010) para farinha de batata-doce, onde observou-se um teor de 0,50\% a $0,75 \%$, Iwe et al. (2004), com 0,5\% e Borba (2005), em torno de $0,7 \%$. Já Andrade e Martins (2002), para fécula de batata-doce comum, obtiveram valores de $1,11 \%$ de lipídeos.

Portanto, os valores de lipídeos encontrados no presente estudo, para a batata-doce comum e biofortificada, foram superiores aos descritos pelos autores citados acima. Este resultado pode acontecer pela influência do solo e clima na produção agrícola das batatas estudadas.

Rodrigues-Amaya et al. (2011) caracterizando resíduos de batata-doce, com base na matéria seca, encontraram um teor de extrato etéreo de 1,73\%, valor superior ao encontrado nesse estudo para batata-doce biofortificada (1,09\%). Estes resultados superiores podem está relacionado ao solvente 
utilizado na extração de compostos orgânicos apolares. Rodrigues-Amaya et al. (2011), utilizou 0 éter etílico e em nossa metodologia utilizamos 0 hexano, sendo que os dois solvente são utilizados para extrair composto orgânicos apalores como por exemplo o $\beta$-caroteno.

Os valores encontrados para 0 teor de lipídeos total, desta pesquisa, encontram-se altos se comparados com os referidos por Leonel e
Cereda.,(2002) e Rogério e Leonel (2002), que referem valores entre $0,3 \%$ e $0,8 \%$.

Já em relação aos carboidratos, observou-se 0 inverso, em que a batata-doce comum apresentou percentual de $15,6 \%$, enquanto a biofortificada (polpa alaranjada), 13,58\% (Figura 5). Valores estes, superiores aos encontrados por Hacineaza et al. (2010) para cultivares de polpa alaranjada e polpa amarela, $7,65 \%$ e $8,7 \%$, respectivamente.

Figura 5. Teor de carboidratos através de média e desvio-padrão das amostras analisadas de batata-doce comum e biofortificada.

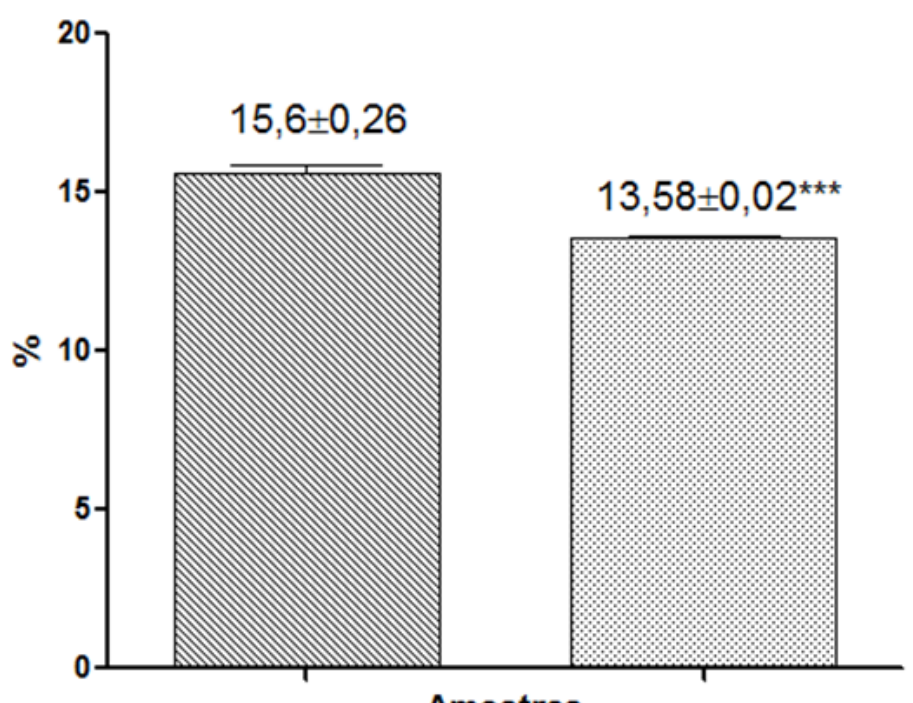

Amostras
Batata Comum

Batata Biofortificada

${ }^{* \star *} \mathrm{p}=0,0002$

Nos estudos realizados por Fontes et al. (2012), foram encontrados valores em torno de $18,38 \%$ de carboidratos na batata-doce comum, porcentagem superior à encontrada neste estudo para ambas batatas.

As diferenças encontradas na caracterização química das batatas-doces comum e biofortificadas in natura podem ser explicadas pela dependência destas em relação à variedade, tipo de solo, adubação, tempo de cultivo e época de colheita (Carvalho et al., 1982).

Os resultados encontrados para lipídeos e carboidratos da batata-doce biofortificada, demonstram que ela é melhor para ser consumida do que a batata-doce comum, pois possui um maior teor de lipídeos e pode ser denominada como alimento de baixo índice glicêmico.

\section{Determinação do pH}

Foram encontrados valores de $\mathrm{pH}$ semelhantes para ambas as batatas-doces, em torno de 5 e 6 , denotando caráter levemente ácido das amostras analisadas conforme Tabela 1.

Tabela 1. pH das amostras de batata-doce comum e biofortificada realizada em triplicata

\begin{tabular}{ccc}
\hline & $\begin{array}{c}\text { Batata Doce - Comum } \\
(\mathrm{pH})\end{array}$ & $\begin{array}{c}\text { Batata Doce- Biofortificada } \\
(\mathrm{pH})\end{array}$ \\
\hline Amostra 1 & Entre 6 e 7 & Entre 5 e 6 \\
Amostra 2 & Entre 5 e 6 & Entre 5 e 6 \\
Amostra 3 & Entre 5 e 6 & Entre 5 e 6 \\
\hline
\end{tabular}


As amostras de batata-doce comum apresentaram uma faixa de $\mathrm{pH}$ próxima a encontrada por Silva (2010) e Andrade e Martins (2002) para farinha da batata-doce, 5,4 e 5,7, respectivamente. De acordo com Giori (2010), o pH ácido interfere diretamente no processo de isomerização das ligações duplas conjugadas, ou seja, é quando o carotenoide perde sua configuração original trans e adquire a forma cis acarretando a perda da intensidade da cor e da atividade pró-vitamina $\mathrm{A}$.

A análise do $\mathrm{pH}$ é de grande relevância por se tratar de um parâmetro que interfere diretamente na qualidade do alimento, ainda mais se tratando de uma variedade peculiar de batata-doce, gerando dúvidas em torno do processo pelo qual é desenvolvida se este pode ou não acarretar danos e descompensações em suas características básicas.

Através desta análise constatamos a manutenção dos valores de $\mathrm{pH}$ esperados para esse tipo de alimento, ou seja, apesar de ser uma cultivar diferenciada não apresentou diferenças em relação ao potencial hidrogeniônico encontrado na batatadoce comum. Além disso, as amostras apresentaram valores próximos à neutralidade, ou seja, faixa de $\mathrm{pH}$ indicada para batata-doce. Pois quanto mais ácido for 0 pH maior será a velocidade de processos de oxidação provocando sua despigmentação e perda da função pró-vitamínica A.

Desta forma evidencia-se que a batata-doce biofortificada é uma potencial estratégia no combate e controle dos casos de hipovitaminose A para humanos ou animais, por seu alto teor de $\beta$-caroteno, precursor da vitamina $A$, como produto agrícola de boa aceitabilidade e acessível às populações carentes que são os grupos mais vulneráveis para deficiências de micronutrientes.

\section{AGRADECIMENTOS}

Os autores agradecem a Faculdade Santo Agostinho, a Universidade Estadual do Piauí e a Universidade Federal do Piauí, pela realização dos experimentos deste estudo.

\section{REFERÊNCIAS BIBLIOGRÁFICAS}

Ahmed, M.; Akter, S.; Eun, J. B. Effect of pretreatments and drying temperatures on sweet potato flour.
International Journal of Food Science \& Technology, $2010,45,4,726-732$

Alves, R.M.V.; Ito, D.; Carvalho, J.L.V.; Melo, W.F.; Godoy, R.L.O. Estabilidade de farinha de batata-doce biofortificada. Brazilian Journal of Food Technology, 2012, 15, 1, 59-71.

AMMAWATH, W.; MAN, Y. C. A rapid method for determination of commercial $\beta$-carotene in RBD palmolein by Fourier transform infrared spectroscopy. Asian Journal of Food and Agro-Industry, 2010, 3, 4, 443-452.

Andrade, R.L.P.; Martins, J.F.P. Influência da adição da fécula de batata-doce (Ipomoea batatas $L$.) sobre a viscosidade do permeado de soro de queijo. Ciência e Tecnologia de Alimentos, 2002, 22, 3, 249-253.

Baranska, M.; Schütze, W.; Schulz, H. Determination of lycopene and $\beta$-carotene content in tomato fruits and related products: Comparison of FT-raman, ATR-IR, and NIR spectroscopy. Analytical Chemistry, 2006, 78, 24, 8456-8461.

Berni, P.R.A.; Chitchumroonchokchai, C.; Brazaca, S.G.C.; Failla, M.L. Bioaccessibility of $\beta$-Carotene in Orange Fleshed Sweet Potato cooked according to home styles compared to highly processed baby foods. Nutrire, 2014, 39, 24-24.

Borba, A.M. Efeitos de alguns parâmetros operacionais nas características físicas, físicoquímicas, e funcionais de extrusados de farinha de batata-doce (Ipomoea batatas). Tese de mestrado em Ciência e Tecnologia de Alimentos - Escola Superior de Agricultura Luiz de Queiroz. São Paulo, 2005.

Britton, $G$. Structure and properties of carotenoids in relation to function. The FASEB Journal, 1995, 9, 15, 1551-1558.

Carvalho, J.L.V.; Nutti, M.R. Biofortificação de produtos agrícolas para nutrição humana. In: REUNIÃO ANUAL DA SBPC, 64., 2012, São Luís. Ciência, cultura e saberes tradicionais para enfrentar a pobreza. São Luís: SBPC: UFMA, 2012. 
Carvalho, M. P.M.; Moura, L.L.; Sturn, A.P. Influência de adubação na produção e na proteína de batata-doce. EMBRAPA. Centro de Tecnologia Agrícola e Alimentar - CTTA. № 2, p.13. Março 1982.

Cereda, M.P.; Wosiacki, G.; Conceição, F.D.A. Características físico-químicas e reológicas de cultivares de batata doce (Ipomoea batatas). Ciência e Tecnologia de Alimentos,1985, 5, 61-70.

Embrapa-Empresa Brasileira de Pesquisa Agropecuária, Batata Doce Beauregard A Batata Vitaminada. 2010. Centro Nacional de Pesquisa de Hortaliças. Disponível em: < https://www.embrapa.br/busca-de-produtosprocessos-e-servicos/-/produto-servico/602/batata-doce--beauregard > Acesso em: 03 de outubro de 2015.

Fontes, L.C B.; Sivi, T.C.; Ramos, K.K.; Queiroz, F.P.C. Efeito das condições operacionais no processo de desidratação osmótica de batata-doce. Revista Brasileira de Produtos Agroindustriais, 2012, 14, 1, 1-14.

Freitas, G.M.; Bueno, G.S.; Ferreira, J.F.; Filho, T.H.G.; Canciam, C.A. Elaboração e análise sensorial de doce de batata-doce com casca (Ipomoea batatas L.). In: V Semana de Tecnologia em Alimentos, 2006. Ponta Grossa-MG. Anais. V Semana de Tecnologia em Alimentos. Ponta Grossa, 2007, 2, 21-25.

Giori, F.P. Adaptação de Metodologia de Digestão In Vitro e Determinação da Bioacessibilidade In Vitro de $\beta$-Caroteno em Três Variedades de Batata Doce de Polpa Alaranjada. Seropédica- RJ, 2010, 68 p. Dissertação - Instituto de Tecnologia, Universidade Federal Rural do Rio De Janeiro.

Hacineaza E.; Vasanthakaalam, H.; Ndirigwe J.; Mukwantali, C. Comparative study on the ß-carotene content and its retention in yellow and orange fleshed Sweet Potato flours. Association for Strengthening Agricultural Research in Eastern and Central Africa, 2010. 5-8.

Hotz, C.; Mcclafferty, B. From harvest to health: Challenges for developing biofortified staple foods and determining their impacton micronutrient status. Food and Nutrition Bulletin, 2007, 28, 2, 271-279.
Instituto Adolfo Lutz. Normas analíticas do Instituto Adolfo Lutz. Métodos químicos e físicos para análise de alimentos. v. 1. 3 ed. São Paulo: Instituto Adolfo Lutz; 1985.

Iwe, M.O.; Van-Zuilichem, D.J.; Stolp, W.; Ngoddy, P. Effect of extrusion cooking of soy-sweet potato mistures on available Lysine content and browning index of extrudates. Journal of Food Engeineering, 2004, 62, 2, 143-150.

Leonel, M.; Cereda, M. P. Caracterização físico-química de algumas tuberosas amiláceas. Ciência e Tecnologia Alimentar. 2002, 22, 1, 65-69.

Leonel, M.; Cereda, M.P. Avaliação da concentração de pectinase no processo de hidrólise-sacarificação do farelo de mandioca para obtenção de etanol. Ciências e Tecnologia de Alimentos, 2000, 20,2, 3-35.

Melo, W.F.; Silva, J.B.C.; Moita, A.W. Avaliação da produtividade de clones de batata-doce ricos em provitamina Ajunto a agricultores familiares. Horticultura Brasileira, 2010, 28, 2, S2302-S2306.

Mirasse, J.J. O consumo de batata-doce de polpa alaranjada entre famílias rurais do nordeste de Moçambique: um estudo sobre percepções de comida e Segurança Alimentar na província de Nampula. Universidade Federal do Rio Grande do Sul, Porto Alegre, UFRGS, 2010, 177p. Dissertação-Mestrado.

Nascimento, K.O.; Rocha, D.G.C.M.; Da Silva, E.B.; Barbosa-Junior, J. L.; Barbosa, M.I.M.J; Nascimento, C.O. Caracterização química e informação nutricional de fécula de batata-doce (Ipomoea batatas L.) orgânica e biofortificada. Revista Verde de Agroecologia e Desenvolvimento Sustentável, 2013, 8, 1, 132-138.

Nunes, M.U.C.; Jesus, A.F.; Lima, I.S.; Santos, L.S.; Cruz, D.P. Produtividade de genótipos de batata-doce com diferentes colorações de raízes em cultivo orgânico. Horticultura Brasileira, 2012, 30, 2, S5542-S5548.

Pavia, D.L.; Lampman, G.M.; Kriz, G.S.; Vyvyan, J.R. Introdução a Espectroscopia. $4^{a}$ ed. São Paulo: Cencage Learning, 2010. 
Rodriguez-Amaya, D.B.; Kimura, M.; Amaya-Farfan, J. Fontes Brasileiras de carotenoides: Tabela Brasileira de composição de carotenoides em alimentos. Ministério do Meio Ambiente, Brasília, 2008.

Rodriguez-Amaya, D. B.; Nutti, M. R.; Carvalho, J. L. V. Carotenoids of sweet potato, cassava, and maize and their use in bread and flour fortification. In: Preedy, R. R.; Watson, R. R.; Patel, V. B. (Eds.). Flour and breads and their fortification in health and disease prevention. London; Burlington; San Diego: Academic Press; Elsevier, 2011. chap. 28, 301-311.

Rogério, W.; Leonel, M. Efeitos da espessura das fatias e pré-cozimento na qualidade de salgadinhos fritos (chips) de tuberosas tropicais. Instituto de Biociências UNESP. 2002, 15, 2, 131-137.

Silva, J.B.C.; Lopes, C.A.; Magalhães, J.S. Cultura da batata doce. Brasília: Embrapa Hortaliças, 2004.
Silva, J.B.C., Silva, E.M.M., Carvalho, P.G.B., Salvador, L., Rangel, C. N., Leastro, D.A., Carvalho, J.L.V., Nutti, M., R. Batata-doce biofortificada: alimento popular com alto teor de pró-vitamina A. In: Simpósio sobre Inovação e Criatividade Científica na Embrapa, 1, 2008, Brasilia.

Silva, J.B.C.; Melo, W.F.; Buso, J.A.; Nutti, M.R.; Carvalho, P.G.B.; Carvalho, J.L.V.; Nunes, M.U.C.; Farias, A. Beauregard: cultivar testada e indicada de batata-doce. Brasília, DF: Embrapa Hortaliças, 2010.

Stevens, R.; Winter-Nelson, A. Consumer acceptance of provitamin A - biofortified maize in Maputo, Mozambique. Food Policy, 2008, 33, 4, 341-351.

Zeraik, M.L.; Yariwake, J.H. Extração de $\beta$-caroteno de cenouras: uma proposta para disciplinas experimentais de química. Química Nova, 2008, 31, 5, 1259-1262. 